\title{
STRUCTURAL AND EXTREMAL PROPERTIES OF THE HOT ROLLING BATCHES PRECEDENCE GRAPH
}

\author{
Anton A. Berezin ${ }^{a}$, Svetlana I. Leonova $^{b}$, Igor A. Vakula $^{c}$ \\ Krasovskii Institute of Mathematics and Mechanics, \\ Ural Branch of the Russian Academy of Sciences, Ekaterinburg, Russia \\ ${ }^{a}$ berezinant@gmail.com, \\ ${ }^{b}$ leonova.svetlana.111@yandex.ru, \\ ${ }^{c}$ igor.vakula@gmail.com
}

\begin{abstract}
In this paper we study structural properties and properties of maximal paths of the hot rolling batches precedence graph. The hot rolling batches precedence graph arises in the problem of planning and scheduling of a hot strip mill load. Slab batches are selected and sequenced in turns. Basic technological restrictions on batch sequencing in turns are represented by the rolling batches precedence graph. Some fundamental structural properties of this graphs are stated such as the local block structure and the maximal paths structure. Motivation and overview of the result application potential are also provided.

Key words: Hot rolling planning, Precedence graph, Graph structure, Local block structure, Maximal paths.
\end{abstract}

\section{Introduction}

Current state of global steel market forces iron-steel plants to investment in product quality improving, operational cost reduction, improvement of customer service. One of the ways to achieve these improvements is development of planning and scheduling systems. Production planning starts from orders and order plan. It determines the amount of steel products of different kind to be produced together with real orders to be done. Shop floor planning includes planning of all production stages starting from heat and cast plans up to cold rolling, polymer coating and etc. Shop floor plan serves to fulfil order plan. It incorporates production and order constraints and requirements. Review [8] describes in detail different practical aspects of planning and scheduling in steel production. One of the emphasizes in this review is the integrated cast-iron and hot rolling planning and processing. In operations research hot rolling production planning problems are known as hot rolling unit planning problems (HRUPPs) when slabs are not batched and inserted in a turn and hot rolling batch planning problems (HRBPPs) [10] where slabs are to be batched and form one or several turns. Problem with predefined batches with equal size slabs can be considered as HRUPP. In this paper we study the structure of geometrical sequencing constraints that are common for HRUPPs and HRBPPs.

At the steel plant at first production stage raw materials such as iron ore and coal are transformed into liquid iron in the blast furnace, then into liquid steel in the melt shop. A fusion is formed by all steel contained in a steel ladle. This liquid steel is usually considered having homogeneous chemical composition. At the next stage liquid steel is continuously casted into solid large steel slabs. Slabs obtained from one fusion and having the same geometrical dimensions are (usually) collected into a batch. In this considered case batches are predefined. At hot strip mill solid large steel slabs are rolled into coils by batches. To ensure the quality of the coils, the working rollers are replaced after a certain length of coils has been rolled. The slabs (coils) collected in batches between two replacements of working rollers are referred to as a hot rolling turn. Production planning and scheduling for hot strip mill are done by planning turns with simultaneous definition of production time windows for every batch (scheduling). To plan a turn (several turns) one should select which batches should be chosen to form a turn(s) and sequence them with respect 
to technological restrictions, their availability in time, keeping productivity of hot strip mill at given level with taking into account the need for coils of different chemical composition and geometrical dimensions for shipment or further processing at cold strip mill and so forth to execute of production orders. Thus there are four basic types of conditions: sequencing conditions, batching conditions, quantity conditions and time conditions. Sequencing conditions describe how units of production can be sequenced in one turn at rolling mill due to technological restrictions. Batching conditions determine the possibilities to create batches from slabs. Batching conditions come from casting production stage depending on chemical composition, sometimes from hot rolling stage depending on unit heating and etc. Quantity conditions define needed amounts (total weight) of slabs with different chemical composition and geometrical dimensions, total weight of a single turn, orders fulfillment and so on. Time conditions contain rolling ready constraints (lower time-window constraints) and customer order readiness conditions. Depending on the situation a part of these conditions can be formulated as constraints and some as optimization criteria. Problems with various constraints can be found in $[3,5,7,9]$.

Starting from Balas $[1,2]$ HRUPPs are formulated as TSPs (PCTSP or other modifications of TSP) where all slabs must be combined in a turn. In PCTSP sequencing conditions are reflected in transition costs between slabs and quantity conditions are reflected by value of nodes (prizes), the total prize for trip minus total cost of transitions is to be minimized. In practice many transitions are impossible and in this setting they must be encoded by infinite weights on corresponding arcs. Sequencing conditions in hot rolling mainly come from slabs geometry width and thickness (or gauge), hardness of slabs steel. Big jumps in thickness and width, increase of width from previous slab to next are penalized. HRBPPs are considered as modifications of VRP, where one or several turns must be scheduled and every turn is the node sequence of a correspondent vehicle. In this case sequencing constraints are again defined by penalties for transitions between batches represented by nodes (by geometry and hardness of slabs in batches). Here quantity constraints are represented by prizes (PCVRP). Time constraints are also considered, as an example one can consider VRPTW [5].

Sequencing either in TSP or VRP setting is generally a hard task. Various authors describe complex integer programming problems for considered extensions of TSP and VRP. The classical integer programming formulation of TSP (analogously for VRP) uses $0-1$ variables $x_{i j}$ to define that slab (batch) $i$ is in place $j$ or in alternative that slab (batch) $j$ is immediately after slab $i$. This leads to very hard problems when hundreds of slabs should be planned to turns. Almost no reducing technics basing on study of structure of sequencing technological constraints are known to authors of this paper. We suppose that this reduction is entrusted to solvers or algorithms. The value of work in different publications is often measured by comparing computational results with results of solving same tasks by human experts. Their authors sometimes even separately mention that no optimality can be achieved in reasonable time in view of hundreds slabs being at income. Here we introduce the HRUPPs setting where sequencing is formalized as a constraint coming from practical tasks at one of the biggest steel productions in Russia (JSC MMK) and study the structure of corresponding precedence graphs. The results as it is shown here can be used to drastically reduce the amount of 0-1 variables in integer programming formulation of considered sequencing problem in comparison with classical approach.

\section{Definitions, tasks and results}

As stated in previous section we consider a HRUPP with predefined batching: a turn is a sequence of given batches consisting of slabs. Every slab is rolled into a coil. All slabs in a batch are having same width, thickness and length and are rolled into coils of same geometrical dimensions. All coils rolled from slabs from one batch are again a batch. Further we will identify slab batches and corresponding coil batches.

Let $P$ be the set of all batches, $|P| \in \mathbb{N}$. Then denote by 
- $w: P \rightarrow \mathbb{R}^{+}$the width function for coils in a batch, by $w_{p}$ we denote the value of $w$ at $p \in P$, further we refer to $w_{p}$ as width of batch $p$;

- $t: P \rightarrow \mathbb{R}^{+}$the thickness function coils in a batch, the value of $t$ at $p \in P$ we denote $t_{p}$, further we refer to $t_{p}$ as thickness of batch $p$;

- $l: P \rightarrow \mathbb{R}^{+}$the weighting function, defining the usefulness of a batch;

- $r: \mathbb{R}^{+} \rightarrow \mathbb{R}^{+}$the monotonically nondecreasing function, defining maximal difference in the thickness between two consecutive batches in turn;

- $\delta \in \mathbb{R}^{+}$the value defining maximal difference in width for two consecutive batches.

The precedence constraint for batches is defined in the following way: a batch $q$ can immediately follow a batch $p$ in a turn if an only if

1. constraint "thickness transition": $\left|t_{p}-t_{q}\right| \leq \min \left\{r\left(t_{p}\right), r\left(t_{q}\right)\right\}$;

2. constraint "width transition": $0 \leq w_{p}-w_{q} \leq \delta$.

Let $G$ be an directed graph with node set $P$ and arc set $E \subseteq P \times P$ such that $p q \in E$ if and only if batches $p$ and $q$ are different and $q$ can immediately follow $p$ in a turn.

The set of all (technologically permissible) turns is equal to $\mathbb{B}(G)$ the set of all paths in $G$ (chains without repeated vertices or edges).

Let $p$ be an arbitrary node in $G$. And let

- $W=\left\{w_{p} \mid p \in P\right\}$

- $P_{w}=\left\{p \mid w_{p}=w\right\}$, where $w \in W$,

- $P_{w^{ \pm}}=\left\{p \in P \mid w-10 \leq w_{p} \leq w+10\right\}$,

- $G_{w}$ be the graph induced by $P_{w}$,

- $P(M)$ be the set of all vertices of subgraph $M$ of graph $G$.

For $w \in W$ consider graph $G_{w}$. It is easy to see that $p$ and $q$ are connected by arc $p q$ if and only if they are connected by $\operatorname{arc} q p$. Let $G_{w}^{0}$ be an ordinary graph with node set $P_{w}$. Two nodes $p$ and $q$ of graph $G_{w}^{o}$ are adjacent if and only if they are connected by arcs $p q$ and $q p$ in graph $G_{w}$. Also let $P(N)$ be the set of all nodes of subgraph $N$ of graph $G_{w}^{0}$.

Precedence constraints described by $G$ are basic sequencing conditions.

To show the reader optimization tasks behind this theoretical work we formulate two formal optimization problems that are close to real world settings.

For a simple path $z$ by $l(z)$ denote the weight of $z$ :

$$
l(z) \triangleq \sum_{p \in P(z)} l(p) .
$$

The first problem (introduced in [6]) is to find in $\mathbb{B}$ a path of maximal weight:

$$
l(z) \rightarrow \max , \quad z \in \mathbb{B}(G) .
$$

To formulate the second problem, first we introduce $F=\left(f_{1}, f_{2}, \ldots, f_{s}\right)$ the set of all production flows. Every flow in practice is formed by all batches that have the same route or destination shop and (or) finished product type. The notion of flow is used to balance the amounts of semiproducts that are produced at previous stage and used as material at next stages. For example hot rolling produces coils for further cold rolling and coating operations. The problem of balancing flows at 
hot rolling stage is to produce hot rolled coals of different flows to ensure stable load of equipment at further production stages at consequent shops. Every hot rolling batch belongs to a single flow.

In production planning it is a task of medium-term planning systems and human experts to provide a flow tasks that should be fullfilled at short-term planning stage so far as this is possible. Particulary, it concerns hot rolling turn planning:

- $f: P \rightarrow F-$ product type function, defining product flow for every batch,

- $\mu: F \rightarrow \mathbb{R}^{+}$— the flow task, the desired total weight of coils for every flow that should be produced in planned turn.

The second problem is to find in $\mathbb{B}$ a simple path $z$ with the flow distribution as close to aim distribution $\mu$ as it is possible. Let

$$
d_{f}(z)=\sum_{f\left(p_{j}\right)=f_{i}, p_{j} \in z} l\left(p_{j}\right) / \sum_{p_{j} \in z} l\left(p_{j}\right) .
$$

As an optimization setting we can consider one of the following or any other relevant:

$$
\sum_{i=1}^{s}\left|\mu\left(f_{i}\right)-d_{f}(z)\right| \rightarrow \min , \quad p_{j} \in z,
$$

or

$$
\max \left|\mu\left(f_{i}\right)-d_{f}(z)\right| \rightarrow \min , \quad p_{j} \in z .
$$

It can be shown (not the aim of this paper) that both problems are $N P$-hard for general graphs. In a separate work (a currently submitted paper) authors introduce an algorithm of time complexity $o\left(n^{3}\right)$ for the first problem. During this study and further investigation of the second problem several results about structural properties of batch precedence graphs were established. Here we prove technical results that generalize previous structural results. They enlighten the structure of batch precedence graphs and as mentioned before are considered useful for further investigation. As a main result of this work we introduce the following.

Theorem 1. Let $H$ be a maximal under inclusion simple path in $G, w \in W$. Then for every block $B$ of graph $G_{w}^{0}$ either $H$ contains all nodes from $B$, or only one, or $H$ and $B$ have no common nodes.

\section{Blocks in $G_{w}^{0}$}

We remind the following definitions (see [4, Ch. III] for definitions and main results on blocks). A cut node of simple graph is a node such that it's removal from graph leads to increase of number of connected components. A nonseparable graph is a nontrivial graph containing no cut nodes. A block in graph is a maximal nonseparable subgraph.

We say that node $p$ is to the left of the node $q$ if $t_{p}<t_{q}$. Similarly we define notions is not to the left, is not to the right, is to the right. We say that node $p$ is between nodes $q_{1}$ and $q_{2}$, if it is not to left of one of them and not to the right of another.

Let $w \in W$. For $G_{w}^{0}$ and its subgraphs writing its node set as $\left\{p_{1}, p_{2}, \ldots, p_{k}\right\}$ with $k \in \mathbb{N}$ we will always mean that they are ordered by growth of values of $t$, i.e $\forall i \in \overline{1, k-1}: \quad t_{p_{i}} \leq t_{p_{i+1}}$.

Lemma 1 (Nonseparability criterion). Let $B$ be a subgraph of graph $G_{w}^{0}, P(B)=\left\{p_{1}, p_{2}, \ldots, p_{k}\right\}$, where $k \geq 3$. Then the following statements are equivalent: 
1. B is connected and nonseparable;

2. $\forall i \in \overline{2, k-1}: \quad p_{i-1} p_{i+1} \in E$.

P r o o f. $(1 \Rightarrow 2)$ Consider a nonseparable subgraph $B$ of graph $G_{w}^{0}$. Next we show that for every $i$ in $\overline{2, k-1}$ there exists an edge $p_{i-1} p_{i+1}$. Suppose that there exists $j$ in $\overline{2, k-1}$ such that $p_{j-1} p_{j+1} \notin E$. Then

for all $l$ such that $l<j-1$ we have $p_{l} p_{j+1} \notin E$ because $t_{p_{j+1}}-t_{p_{j-1}} \leq t_{p_{j+1}}-t_{p_{l}}$, and similarly for all $m>j+1$ we have $p_{j-1} p_{m} \notin E$, because $t_{p_{j+1}}-t_{p_{j-1}} \leq t_{p_{m}}-t_{p_{j-1}}$.

This implies that deleting node $p_{j}$ from $B$ leads to that every node with values of $t$ less, than $t_{p_{j}}$ would be unadjacent with nodes, having value of $t$ greater, than $t_{p_{j}}$. Considering that $k \geq 3$ we get that subgraph $B-p_{j}$ is disconnected. Therefore $p_{j}$ is a cut node of $B$, which contradicts the choice of $B$. Thus $\forall i \in \overline{2, k-1}: \quad p_{i-1} p_{i+1} \in E$.

$(1 \Leftarrow 2)$ Consider subgraph $B$ of $G_{w}^{0}$. By condition 2 for all $i$ in $\overline{2, k-1}: \quad p_{i-1} p_{i+1} \in E$, it follows, that $\forall i \in \overline{1, k-1} p_{i} p_{i+1} \in E$, because $t_{p_{i+1}}-t_{p_{i}} \leq t_{p_{i+1}}-t_{p_{i-1}}$. Therefore, $B$ is connected.

Next we prove that $B$ does not contain cut nodes. Consider arbitrary node $p_{j}$ in $B$. If $j=1$ or $j=k$ subgraph $B-p_{j}$ is obviously connected, for other values of $j$ nodes $p_{j-1}$ and $p_{j+1}$ are defined and adjacent, thus $B-p_{j}$ is also connected. Therefore by deleting any node in $B$ one gets a connected graph, consequently $B$ is nonseparable.

Proposition 1. In cases $|P(B)|=1$ and $|P(B)|=2$ subgraph $B$ is nonseparable in any case.

Lemma 2 (Maximality criterion for nonseparable subgraph). Let $B$ be a connected nonseparable subgraph of $G_{w}^{0}, P(B)=\left\{p_{1}, p_{2}, \ldots, p_{k}\right\}, k \in \mathbb{N}$. Then the following conditions are equivalent:

1. $B$ is maximal nonseparable subgraph in $G_{w}^{0}$ (block).

2. Either $P(B)=\left\{p_{1}\right\}$, where $p_{1}$ is an isolated node in $G_{w}^{0}$, or for any $p$ in $P_{w} \backslash P(B)$ either $t_{p}<t_{p_{1}}$ and $p p_{2} \notin E$ or $t_{p}>t_{p_{k}}$ and $p_{k-1} p \notin E$.

P r o o f. $\quad(1 \Rightarrow 2)$ Let $B$ be a maximal (under inclusion) nonseparable subgraph of $G_{w}^{0}$. Consider a node $p \in P_{w} \backslash P(B)$.

Let $k=1$. Maximality of $B$ implies $p p_{1} \notin E$, therefore $p_{1}$ is an isolated node.

Let $k>1$. Consider subgraph $B^{\prime}$, induced by $P(B) \cup\{p\}$. From maximality of $B$ it follows that $B^{\prime}$ is separable.

Let $t_{p} \in\left[t_{p_{1}}, t_{p_{k}}\right]$.

Let $k=2$. Connectivity of $B$ implies that $p_{1} p_{2} \in E$, therefore $p_{1} p \in E$ and $p p_{2} \in E$. Consequently, by Lemma 1 subgraph $B^{\prime}$ is nonseparable, which contradicts maximality of $B$.

Let $k>2$. Then there exists $i \in \overline{1, k-1}$ such, that $t_{p} \in\left[t_{p_{i}}, t_{p_{i+1}}\right]$.

If $i \in \overline{2, k-2}$, then by Lemma 1 we have $p_{i-1} p_{i+1} \in E$ and $p_{i} p_{i+2} \in E$. This in its turn implies $p_{i} p_{i+1} \in E$. Considering the existence of edges $p_{i-1} p_{i+1}, p_{i} p_{i+2}, p_{i} p_{i+1}$ we get, that $p_{i-1} p, p_{i} p, p p_{i+1}$ and $p p_{i+2}$ also exist, thus by Lemma 1 graph $B^{\prime}$ is a nonseparable subgraph of graph $G_{w}^{0}$, which contradicts the maximality of $B$.

If $i=1$, then by Lemma 1 we get $p_{1} p_{3} \in E$. This implies the existence of edges $p_{1} p, p p_{2}$ $p p_{3}$, consequently by Lemma 1 subgraph $B^{\prime}$ is a nonseparable subgraph of graph $G_{w}^{0}$, which again contradicts the maximality of $B$.

The case $i=k-1$ is treated similarly to case $i=1$.

Now it is proved that for any $k>1$ assumption $t_{p} \in\left[t_{p_{1}}, t_{p_{k}}\right]$ leads to a contradiction. Therefore we can assume, that $t_{p}<t_{p_{1}}$ or $t_{p}>t_{p_{k}}$.

Let $t_{p}<t_{p_{1}}$. If $p p_{2} \in E$ then by Lemma 1 subgraph $B^{\prime}$ is nonseparable. Similarly if $t_{p}>t_{p_{k}}$ and $p_{k-1} p \in E$ again subgraph $B^{\prime}$ is nonseparable. Both contradict the maximality of $B$.

Thus, if $p \in P_{w} \backslash P(B)$ and $k>1$ then either $t_{p}<t_{1}$ and $p p_{2} \notin E$ or $t_{p}>t_{k}$ and $p_{k-1} p \notin E$. 
$(1 \Leftarrow 2)$ Choose any $p \in P_{w} \backslash P(B)$ and consider subgraph $B^{\prime}$ of $G_{w}^{0}$, induced by set $P(B) \cup\{p\}$.

Condition $P(B)=\left\{p_{1}\right\}$, where $p_{1}$ is an isolated node means, that $B^{\prime}$ is not connected, consequently, considering $p$ is arbitrary we get that $B$ is maximal nonseparable subgraph of $G_{w}^{0}$.

Condition $\forall p \in P_{w} \backslash P(B)$ either $t_{p}<t_{p_{1}}$ and $p p_{2} \notin E$ or $t_{p}>t_{p_{k}}$ and $p_{k-1} p \notin E$ means, that in any case $B^{\prime}$ does not satisfy condition 2 of Lemma 1, thus subgraph $B^{\prime}$ is separable, therefore $B$ is maximal nonseparable subgraph of $G_{w}^{0}$.

\section{Relations between paths in $G$ and blocks of $G_{w}^{0}$}

Let $H_{1}=p_{1} p_{2} \ldots p_{n}$ and $H_{2}=q_{1} q_{2} \ldots q_{m}$ be two arbitrary simple paths. If $H_{1}$ and $H_{2}$ do not have common nodes and $p_{n} q_{1} \in E$, then by sum of $H_{1}$ and $H_{2}$ we call a path $H=p_{1} \ldots p_{n} q_{1} \ldots q_{m}$. If $H_{1}$ and $H_{2}$ have a single node in common and it is $p_{n}=q_{1}$, then by sum of $H_{1}$ and $H_{2}$ we call a path $H=p_{1} \ldots p_{n} q_{2} \ldots q_{m}$. If none of given conditions is satisfied then the sum of paths is not defined. Lets denote the sum as follows $H=H_{1}+H_{2}$. It is clear, that the sum of chains is associative and non-commutative operation.

Lemma 3. Let $B$ be a block of graph $G_{w}^{o}, s, e \in P(B), t_{s} \leq t_{e}$. Then the following assertions hold:

1. If there exists at least one node $q \in P(B)$, distinct from $s$ and e and such, that $t_{s} \leq t_{q} \leq t_{e}$, then there exists a $(s, e)$-path, constaining all nodes of block.

2. If for every node $q \in P(B)$, distinct from $s$ and $e$ we have $t_{s} \leq t_{q}$, then there exists an $(s, e)$-path, containing all nodes of the block.

3. If for every node $q \in P(B)$, distinct from $s$ and $e$ we have $t_{q} \leq t_{e}$, then there exists an $(s, e)$-path, containing all nodes of the block.

P r o o f. Since $G_{w}^{o}$ is an undirected graph, then without loss of generality we can consider only the case $t_{s} \leq t_{e}$.

Let $P(B)=\left(p_{1}, p_{2}, \ldots, p_{i}=s, q_{1}, q_{2}, \ldots, q_{l}, p_{j}=e, p_{j+1}, \ldots p_{k}\right)$. As it was noted earlier nodes of $B$ are listed with nondecreasing values of $t$. Moreover, we claim that $t_{p_{i-1}} \leq t_{s}$ and $t_{p_{j}} \geq t_{e}$.

Construct an $\left(s, q_{1}\right)$-path $H_{1}$ in the following way: use every second node while moving to the left from $q_{1}$ towards $p_{1}$, then use every unused node while moving to the right from $p_{1}$ towards $q_{1}$. More precisely:

$$
H_{1}= \begin{cases}p_{i} p_{i-2} p_{i-4} \ldots p_{2} p_{1} p_{3} p_{5} \ldots p_{i-3} p_{i-1} q_{1}, & \text { if } i \text { is even } \\ p_{i} p_{i-2} p_{i-4} \ldots p_{3} p_{1} p_{2} p_{4} \ldots p_{i-3} p_{i-1} q_{1}, & \text { if } i \text { is odd }\end{cases}
$$

All edges in $H_{1}$ exist in the view of nonseparability of $B$. This is true for the other paths introduced further. $H_{1}$ contains all $p \in P(B)$ having $t_{p}<t_{s}$. This proves 3 if we consider that $q_{1}=e$ and there are no nodes to the right of $e$.

Similarly $\left(q_{l}, e\right)$-path $\mathrm{H}_{2}$ is as follows:

$$
H_{2}= \begin{cases}q_{l} p_{j+1} p_{j+3} \ldots p_{k-2} p_{k} p_{k-1} p_{k-3} \ldots p_{j+4} p_{j+2} p_{j}, & \text { if }(k-j) \text { is even } \\ q_{l} p_{j+1} p_{j+3} \ldots p_{k-1} p_{k} p_{k-2} p_{k-4} \ldots p_{j+4} p_{j+2} p_{j}, & \text { if }(k-j) \text { is odd }\end{cases}
$$

$H_{2}$ contains all nodes $p \in P(B)$ such, that $t_{p}>t_{e}$. This proves 3 if we consider that $q_{1}=s$ and there are no nodes to the left of $s$.

A $\left(q_{1}, q_{l}\right)$-path is $H_{3}=q_{1} q_{2} \ldots q_{l}$.

$H_{3}$ contains all nodes $p \in P(B)$ such, that $t_{s} \leq t_{p} \leq t_{e}$. Then we construct an $(s, e)$-path containing all nodes of $B$ as a sum of $H_{i}: H=H_{1}+H_{3}+H_{2}$.

Lemma 4. Let $H$ be a path in $G$, then for any two consequent nodes $p$ and $q$ in $H, w_{p} \geq w_{q}$. 
P r o o f. The statement of lemma by transitivity follows from that for any arc $p^{\prime} p^{\prime \prime}$ of path $H$ according to width transition constraint $w_{p^{\prime}}-w_{p^{\prime \prime}} \geq 0$ or $w_{p^{\prime}} \geq w_{p^{\prime \prime}}$.

Lemma 5. Let $B$ be a block of graph $G_{w}^{o}, H=q_{1} q_{2} \ldots q_{l}$ be a path in graph $G$. Let $M \subset B$ be a set of all nodes in $B$ contained in $H, m=|M|$. Let $u=\min _{i}\left\{q_{i} \mid q_{i} \in M\right\}$ and $v=\max _{i}\left\{q_{i} \mid q_{i} \in M\right\}$. Then for all $i$ such that $u \leq i \leq v$ node $q_{i}$ is in $M$.

P r o o f. In view of Lemma 4 all nodes in $H$ between $q_{u}$ and $q_{v}$ belong to $G_{w}^{o}$. Let $M=$ $\left\{p_{1}, p_{2}, \ldots, p_{s}\right\}$. Suppose that there exists $j$ such, that $u \leq j \leq v$ and $q_{j} \notin M$. Then by statement 2 of Lemma 2 every node $q_{i}$ not in $M$ lies to the left or to the right of all nodes of $M$. Suppose without a loss of generality that $q_{j}$ is to the left of $M$. Let $q_{l}$ be the first node from $P(H) \cap G_{w}^{0} \backslash M$ in $q_{s} q_{s+1} \ldots q_{e}$ and $q_{m}$ be the last node from $P(H) \cap G_{w}^{0} \backslash M$ in this path. Then they are both connected to nodes from $M$, moreover these nodes from $M$ are distinct. Thus one of $q_{l}$ and $q_{m}$ is connected to $p_{2}$ (and certainly to $p_{1}$ ). Which due to statement 2 of Lemma 2 contradicts the maximality of $B$ as a separable subgraph in $G$.

\section{Proof of Theorem 1}

Consider an arbitrary block $B$ in $G_{w}^{0}$ ( $w$ is arbitrary too). Suppose that $B$ has not less then two common nodes with $H$. To prove theorem it is enough to show that $H$ contains all nodes from $B$ is this case.

In view of Lemma 5 nodes from different blocks from $G_{w_{i}}^{0}$ (for all $w_{i}$ ) do not interleave.

Consider two nodes $s$ and $e$ the entrance and exit of $H$ in $B$, they are distinct in view of simplicity of $H$ and considered case that $|P(B) \cap P(H)| \geq 2$. Without loss of generality we suppose that $t_{s} \leq t_{e}$. From Lemma 3 it follows that in any case that all nodes of $B$ are not left of $s$ or are not right of $e$ or there exists a node between $s$ and $e$ different from them then there exists a path $H^{\prime}$ containing all nodes of $B$ in view of maximality of $H$. This follows $P(B) \subseteq P(H)$. Thus further we can suppose that $B$ contains no nodes between $s$ and $e$ different from them and that there exist nodes to the left of $s$ and to the right of $e$ in $B$. Let $q_{1}=\arg \max _{b \in B: t_{b}<t_{s}} t_{b}$ and $q_{2}=\arg \min _{b \in B: t_{b}>t_{e}} t_{b}$. Since $B$ is a block then by Lemma 1 there exist edges $q_{1}^{-} s$ and $e q_{1}^{+}$as well as edges $q_{1}^{-} e$ and $s q_{1}^{+}$.

If $s$ is the first node in $H$ then consider a path $H^{\prime}$ that starts from $q_{1}$ and ends at $e$ in $B$ and containing all nodes from $B$ which is possible by Lemma 3 and coincides with the path $H$ after $e$. Then $H^{\prime}$ contains $H$ and by maximality of $H$ all nodes of $H^{\prime}$ including those in $B$ contain in $H$. Similarly it is proved that in case when $e$ is the last node in $H$ it follows that $H$ contains all nodes from $B$. Further we assume that $s$ and $e$ are not respectively the first and the last nodes in $H$.

Let $p_{1}$ be a node immediately preceding $s$ in $H$. If edge $p q_{1}$ exists then path $H^{\prime}$ coinciding $H$ up to $p_{1}$ and from $e$ and after $p$ passing through $q_{1}$ then through all nodes in $B$ and exiting out of $B$ in $e$ (exists by Lemma 3) contains $B$ and $H$, thus my maximality of $H$ we have $P(B) \subseteq P\left(H^{\prime}\right) \subseteq P(H)$. Further we assume that $p q_{1}$ does not exist. This implies that $t_{p_{1}}>t_{e}$ because $e$ is adjacent with $q_{1}$ in $G_{w}^{0}$. Therefore $p_{1}$ is adjacent to $q_{2}$ in $G_{w}^{0}$. Similarly it is proved that $p_{2}$ the the next node after $e$ is adjacent to $q_{1}$. Next if we consider path $H^{\prime}$ coinciding with $H$ up to $p_{1}$ and from $p_{2}$, entering in $q_{2}$ in $B$ and exiting at $q_{1}$ and containing all nodes in $B$ (by Lemma 3 it is possible) we again get that nodes of $B$ are contained in $H$ because of maximality of $H$.

\section{Conclusions}

In this paper authors investigated structure of sequencing constraints for a variant formulation of the hot rolling unit planning problem. Practical sequencing constraints were formalized by the precedence graph of rolling batches with turns being simple paths in this precedence graphs. Local (layer) structure and maximal paths of this graph were studied. It was proved that every block 
of special (layered by width) subgraphs and any maximal path intersect by 0,1 or all nodes of a block. These results as it is shown here can drastically reduce the number of $0-1$ variables in integer programming formulation of hot rolling planning problems that can even lead to optimal solutions in practical tasks. Further investigation plan is to complete computational experiments with improved integer programming models on real world data and to report new results on optimality issues with mathematical proof of modifications being used.

\section{REFERENCES}

1. Balas E. The prize collecting travelling salesman problem // Networks, October 1989. Vol. 19. P. 621636.

2. Balas E., Clarence H.M. Combinatorial optimization in steel rolling // Workshop on Combinatorial Optimization in Science and Technology, April, 1991.

3. Cowling P., Rezig W. Integration of continuous caster and hot strip mill planning for steel production // Journal of Scheduling, July-August, 2000. Vol. 3, no. 4. P. 185-208.

4. Harary F. Graph Theory. MA: Addison-Wesley, Reading, 1969. 274 p.

5. Chen X., Wan, W.-S. and Xu, X.-H. Modeling rolling batch planning as vehicle routing problem with time windows // Computers \& Operations Research, 1998. Vol. 25, no. 12. P. 1127-1136.

6. Vakula I.A., Leonova S.I. On building hot rolling turns // Contemporary problems in mathematics and its applications: 45-th International Youth School-Conference: Proceedings. Ekaterinburg: IMM UrB RAS, USU. 2014. P. 164-166. [In Russian]

7. Vakula I.A., Leonova S.I. Hot rolling planning problem // Mathematical programming and applications: XV-th All-Russian Conference: Abstracts. Ekaterinburg: IMM UrB RAS, USU. 2015. P. 77-78. [In Russian]

8. Lixin Tang, Jiyin Liu, Aiying Rong, Zihou Yang. A review of planning and scheduling systems and methods for integrated steel production // European Journal of Operational Research. August 16, 2001. Vol. 133, no. 1. P. 1-20.

9. Xianpeng Wang, Lixin Tang. Integration of batching and scheduling for hot rolling production in the steel industry // The International Journal of Advanced Manufacturing Technology. 2008. Vol. 36. P. 431-441.

10. Shixin Liu. Model and Algorithm for Hot Rolling Batch Planning in Steel Plants // International Journal of Information and Management Sciences. 2010. Vol. 21, no. 3. P. 247-263. 